\title{
Evolution and analysis of COVID-2019 through a fractional mathematical model
}

\author{
M. S. Kiran ${ }^{1}$, Virupaxappa Betageri ${ }^{1}$, D. G. Prakasha ${ }^{2}$, P. Veeresha ${ }^{3}$, and Sunil Kumar ${ }^{4}$ \\ ${ }^{1}$ GM Institute of Technology, Davangere-577006, Karnataka, India \\ ${ }^{2}$ Davangere University \\ ${ }^{3}$ Karnatak University Dharwad \\ ${ }^{4}$ National Institute of Technology Jamshedpur
}

May 6, 2020

\begin{abstract}
Throughout the globe, mankind is in vastly infected situations due to a cruel and destructive virus called coronavirus (COVID19). The pivotal aim of the present investigation is to analyze and examine the evolution of COVID-19 in India with the available data in two cases first from the beginning to 31st March and beginning to 23rd April in order to show its exponential growth in the crucial period. The present situation in India with respect to confirmed, active, recovered and deaths cases have been illustrated with the aid of available data. The species of novel virus and its stages of growth with respect some essential points are presented. The exponential growth of projected virus by the day-to-day base is captured in $2 \mathrm{D}$ plots to predict its developments and identify the needs to control its spread on mankind. Moreover, the SEIR model is considered to present some interesting consequences about COVID-19 within the frame of fractional calculus. A newly proposed technique called q-Homotopy analysis transform method (q-HATM) is hired to find the solution for the nonlinear system portraying projected model and also presented the existence and uniqueness of the obtained results with help of fixed point theory. The behaviour has been captured with respect to fractional order and time.
\end{abstract}

\section{Hosted file}

Manuscript-2020.docx available at https://authorea.com/users/317337/articles/447446evolution-and-analysis-of-covid-2019-through-a-fractional-mathematical-model 
figures/Figure-1/Figure-1-eps-converted-to.pdf 
figures/Figure-2-(a)/Figure-2-(a)-eps-converted-to.pdf 
figures/Figure-2-(b)/Figure-2-(b)-eps-converted-to.pdf 
figures/Figure-2-(c)/Figure-2-(c)-eps-converted-to.pdf 
figures/Figure-2-(d)/Figure-2-(d)-eps-converted-to.pdf 
figures/Figure-3/Figure-3-eps-converted-to.pdf 
figures/Figure-4-(a)/Figure-4-(a)-eps-converted-to.pdf 
figures/Figure-4-(b)/Figure-4-(b)-eps-converted-to.pdf 
figures/Figure-5-(a)/Figure-5-(a)-eps-converted-to.pdf 
figures/Figure-5-(b)/Figure-5-(b)-eps-converted-to.pdf 
figures/Figure-5-(c)/Figure-5-(c)-eps-converted-to.pdf 
figures/Figure-5-(d)/Figure-5-(d)-eps-converted-to.pdf 
figures/Figure-6/Figure-6-eps-converted-to.pdf 
figures/Figure-7-(a)/Figure-7-(a)-eps-converted-to.pdf 
figures/Figure-7-(b)/Figure-7-(b)-eps-converted-to.pdf 
figures/Figure-8-(i)/Figure-8-(i)-eps-converted-to.pdf 
figures/Figure-8-(ii)/Figure-8-(ii)-eps-converted-to.pdf 
figures/Figure-8-(iii)/Figure-8-(iii)-eps-converted-to.pdf 
figures/Figure-8-(iv)/Figure-8-(iv)-eps-converted-to.pdf 